\title{
NOVEDADES EN POLYBOTRYA (FILICALES: DRYOPTERIDACEAE) PARA COSTA RICA
}

\author{
Alexander Fco. Rojas Alvarado \\ Jardín Botánico Lankester. Universidad de Costa Rica. \\ Apdo.1031-7050, Cartago, Costa Rica. alfrojasa@yahoo.com
}

\begin{abstract}
In this paper two new species of Polybotrya are described as new: P. aureisquama and $P$. insularis. Polybotrya aureisquama is different from $P$. alfredii by broader, orange-gold rhizome scales with long ciliate margin, shorter fronds, less differentiated color between blade surfaces and longer axes hairs. Polybotrya insularis is different from $P$. osmundacea by shorter fronds, glabrescent axes, anadromic pinnules in medial pinnae and bipinnate fertile blade. Also, Polybotrya lourteigiana is registered from Costa Rica.
\end{abstract}

Resumen. En este trabajo se describen dos especies nuevas de Polybotrya: P. aureisquama y P. insularis. Polybotrya aureisquama difiere de $P$. alfredii por las escamas del rizoma más anchas, anaranjado-doradas (vs. pardas) y con margen largamente ciliado (vs. entero), frondas más cortas, lámina menos diferenciada en color entre ambas superficies y pelos de los ejes más largos. Polybotrya insularis se distingue de $P$. osmundacea por las frondas estériles más cortas, ejes glabrescentes (no escamosos y dispersamente pelosos), pínnulas anadrómicas en las pinnas medias y frondas fértiles bipinnadas. También, se cita Polybotrya lourteigiana para Costa Rica.

KeY Words: Dryopteridaceae, Polybotrya, new species, new record, Costa Rica.

Polybotrya Willd., es un género caracterizado por frondas fuertemente dimorfas, las frondas fértiles similar a las estériles en la división de la lámina pero el tejido foliar reducido, el rizoma usualmente largamente rastrero y cubierto de escamas, con anatomía única de 5 a 12 meristelas dispuestas circularmente, cada una rodeada con una vaina esclerenquimatosa negra y con numerosas trazas foliares delgadas arqueando entre las meristelas adyacentes (Moran 1987).

En las últimas tres décadas se han descrito varias especies nuevas de Polybotrya Fée para el Neotrópico, entre ellas: Polybotrya crassirhizoma Lellinger, $P$. macbridei Lellinger, $P$. salicifolia Lellinger, $P$. decorata Lellinger y $P$. subelliptica Lellinger (Lellinger 1972); P. lourteigiana Lellinger y $P$. pittieri Lellinger (Lellinger 1977). De las especies anteriores descritas por Lellinger, Moran (1987) sólo reconoce tres y describe diez especies nuevas, $P$. aequatoriana Moran, $P$. alata Moran, $P$. appressa Moran, $P$. attenuata Moran, $P$. gomezii Moran, $P$. hickeyi Moran, $P$. latisquamosa Moran, $P$. puberulenta Moran, P. sessilisora Moran y P. stolzei Moran. El último autor también menciona que Polybotrya caudata Kunze es una especie con un amplio ámbito de distribución y con mucha variabilidad en la pubescencia.

Rojas y Trusty (2004) citan erróneamente a $P$. osmundacea Humb. et Bonpl. ex Willd. para la Isla del Coco, basándose en que los ejemplares colectados en la isla presentan las pinnas basales anádromas y el margen de los segmentos sin tricomas. $P$. caudata se cita para la isla sólo por el espécimen J. Trusty 1426 (CR), en tanto que el resto de las muestras registradas para la isla como cualquiera de estas dos especies, corresponden a una especie nueva descrita aquí como P. insularis A. Rojas. En el caso de P. alfredii Brade, la descripción de Moran $(1987,1995)$ indica que es una especie variable en las escamas del rizoma y división de la lámina, en esta investigación se encontró además que había variación en la forma de las escamas, tamaño los tricomas de los ejes y que existía correlación de varios caracteres, por lo cual se describe a $P$. aureisquama A. Rojas y se registra a $P$. lourteigiana Lellinger para Costa Rica. 
Polybotrya aureisquama A. Rojas, sp. nov.

TIPO: Costa Rica. Alajuela: San Ramón, Reserva Biológica Alberto Manuel Brenes, Sendero Terciopelo, $10^{\circ} 12^{\prime} 45^{\prime \prime} \mathrm{N}, 84^{\circ} 36^{\prime} 25^{\prime \prime} \mathrm{W}, 1000 \mathrm{~m}, 2$ mar 2006, A. Rojas et al. 6986 (Holotipo: CR; Isotipos: K, MO, NY, USJ). FIG. 1 y 2.

A Polybotrya alfredii Brade stipitis squamis aureis (adversus pardales) margine ciliata (adversus integram), frondibus brevioribus, costis costulisque longisetosis (adversus brevipilosas) differt.

Hemiepífitas; rizoma de 0.5-1 cm de diámetro, reptante; escamas del rizoma 7-10 x 1.5-2.5 mm, lanceoladas, doradas a ferrugíneas, levemente bicolores, con el centro pardo a rojizo, opacas, flácidas, margen largamente ciliado (procesos semejantes a pelos); frondas dimorfas, las estériles de 50-70 (-85) cm de largo; estípite $12-20 \mathrm{~cm}$ de largo, pajizo, escamoso en toda su extensión, las escamas hasta $15 \mathrm{~mm}$ de largo, en lo demás similares a las del rizoma, más densas y largas las de la base; lámina estéril 34-50 (-65) x 25-35 (-60) $\mathrm{cm}$, 2-pinnado-pinnatífida a 3-pinnada, deltada, el tejido glabro entre las nervaduras, el envés verde claro (levemente más claro que el haz); pinnas 15-20 x 5-12 cm, catádromas, profundamente incisas hasta el ápice; pínnulas de las pinnas medias 1.5-5 x 0.5-1.8 $\mathrm{cm}$, la base pediculada $1-3 \mathrm{~mm}$; raquis y costas pajizos, glabros en el haz, dispersamente pelosos sólo en el surco dorsal, pelosos en el envés, los tricomas 0.5$1 \mathrm{~mm}$ de largo, dorados a pardo claro; nervaduras libres, simples o bifurcadas, pinnadas en cada segmento y hasta 3 pares; frondas fértiles 3-4-pinnadas, las pínnulas lanceoladas, pinnadas; últimos segmentos fértiles de 1-3 mm de largo, los terminales hasta 5 $\mathrm{mm}$ de largo; esporas normalmente desarrolladas.

DistRIBUCIÓN: En vertiente norte y Caribe de las cordilleras de Costa Rica y en el Pacífico Central a 700$1600 \mathrm{~m}$.

Paratipos: COSTA RICA. Alajuela: Reserva Forestal San Ramón, near of the station, $10^{\circ} 12^{\prime} 40^{\prime \prime} \mathrm{N}$, 84³6'20”'W, 900-1000 m, 12 ene 1991, J. Bittner 253 (CR); Reserva Forestal San Ramón, S of the station, 2-3 $\mathrm{km}$ of the station, $10^{\circ} 12^{\prime} 40^{\prime \prime} \mathrm{N}, 84^{\circ} 36^{\prime} 20^{\prime \prime} \mathrm{W}$, 1200-1400 m, 20 mar 1991, J. Bittner 778 (USJ); Reserva Forestal San Ramón, S of the station, ca. 2 $\mathrm{km}$ in the mountains, $10^{\circ} 12^{\prime} 40^{\prime \prime} \mathrm{N}, 84^{\circ} 36^{\prime} 20^{\prime \prime} \mathrm{W}, 1000$ -
1200 m, 16 abr 1991, J. Bittner 952 (CR, USJ), J. Bittner 956 (CR); Reserva Forestal San Ramón, ca. 2 $\mathrm{km} \mathrm{S}$ of the station, $10^{\circ} 12^{\prime} 40^{\prime \prime} \mathrm{N}, 84^{\circ} 36^{\prime} 20^{\prime \prime} \mathrm{W}, 1000-$ 1100 m, 27 mayo 1991, J. Bittner 1030 (CR); Reserva Forestal San Ramón, trail $2 \mathrm{~km} \mathrm{~N}$ of the field station, $10^{\circ} 12^{\prime} 40^{\prime \prime} \mathrm{N}, 84^{\circ} 36^{\prime} 20^{\prime \prime} \mathrm{W}, 1000 \mathrm{~m}, 21$ oct 1993, J. Bittner \& G. Herrera 2150 (CR, USJ); Reserva San Ramón, Bajos de Jamaical, 70-100 m, 10 may 1985, I. Chacón 1770 (CR); Cantón de San Ramón, Reserva Forestal San Ramón, Cordillera de Tilarán, Sendero Polígono, $10^{\circ} 13^{\prime} 00^{\prime} \mathrm{N}, 84^{\circ} 35^{\prime} 20^{\prime} \mathrm{W}, 800-1000 \mathrm{~m}, 8$ may 1996, E. Fletes et al. 291 (INB, MO); along Río Sarapiquí, upstream from crossing road Colonia Virgen del Socorro, $10^{\circ} 5^{\prime} 24^{\prime \prime} \mathrm{N}, 84^{\circ} 10^{\prime} 12^{\prime}$ W, ca. 740 m, 3 jul 1985, M. Grayum \& B. Hammel 5525 (CR, $\mathrm{MO}$ ); San Carlos, La Fortuna, Finca El Jilguero, Sendero La Lava, Río Aguas Calientes, $0.5 \mathrm{~km}$ aguas arriba en el margen izquierdo, $10^{\circ} 26^{\prime} 35^{\prime \prime} \mathrm{N}$, 8442’20”W, 700 m, 23 nov 1992, G. Herrera 5604 (CR); Cantón de San Ramón, Los Ángeles, Reserva Forestal San Ramón, $1 \mathrm{~km}$ NW de la estación, $10^{\circ} 12^{\prime} 40^{\prime \prime} \mathrm{N}$; 84³6'20”'W, 1000 m, 2 dic 1993, G. Herrera 6717 (CR); 5 km NNW of San Ramón by road, $2.5 \mathrm{~km} \mathrm{~N}$ of Balsa on road to San Lorenzo, $10^{\circ} 22^{\prime} \mathrm{N}, 84^{\circ} 30^{\prime} \mathrm{W}, 1050-1100 \mathrm{~m}, 25 \mathrm{abr}$ 1983, R. Liesner \& E. Judziewicz 14855 (CR, MO); Cantón de San Ramón, Reserva Biológica Alberto Manuel Brenes, Cordillera de Tilarán, Estación San Ramón, $10^{\circ} 13^{\prime} 10^{\prime \prime} \mathrm{N}, 84^{\circ} 36^{\prime} 00^{\prime \prime} \mathrm{W}, 800-1000 \mathrm{~m}, 6$ mayo 1996, M. Moraga et al. 360 (INB); ca. $20 \mathrm{~km} \mathrm{~N}$ of San Ramón, University of San Ramón, Biological Field Station, 1100 m, 17 jul 1983, R. Moran 3214 (CR, MO); Cantón de San Ramón, Reserva Biológica Alberto Manuel Brenes, Sendero Terciopelo, $50 \mathrm{~m}$, de la estación, $10^{\circ} 13^{\prime} 06^{\prime \prime} \mathrm{N}, 84^{\circ} 35^{\prime} 43^{\prime \prime} \mathrm{W}, 900 \mathrm{~m}, 3 \mathrm{jul}$ 2003, R. Moran \& A. Cooley 6723 (CR, USJ); Cantón de Alajuela, Cordillera Central, Sarapiquí, entre Cariblanco y Virgen del Socorro y orillas del Río Sarapiquí, $10^{\circ} 15^{\prime} 25^{\prime \prime} \mathrm{N}, 8^{\circ} 10^{\prime} 20^{\prime \prime} \mathrm{W}, 720-870 \mathrm{~m}, 21$ ago 1994, A. Rojas 1391 (CR, INB); San Ramón, Reserva Biológica Alberto Manuel Brenes, Sendero Heliconias, $10^{\circ} 13^{\prime} 15^{\prime \prime} \mathrm{N}, 84^{\circ} 36^{\prime} 45^{\prime \prime} \mathrm{W}, 1000-1200 \mathrm{~m}$, 5 sept 2006, A. Rojas \& J. Cervantes 7339 (CR, MO, SEL, USJ); San Ramón, Reserva Biológica Alberto Manuel Brenes, Sendero Heliconias, $10^{\circ} 13^{\prime} 15^{\prime \prime} \mathrm{N}$, 84³6'45"W, 1000-1200 m, 1 jun 2006, A. Rojas et al. 7183 (CR, MO, SEL, USJ); Reserva Forestal San 


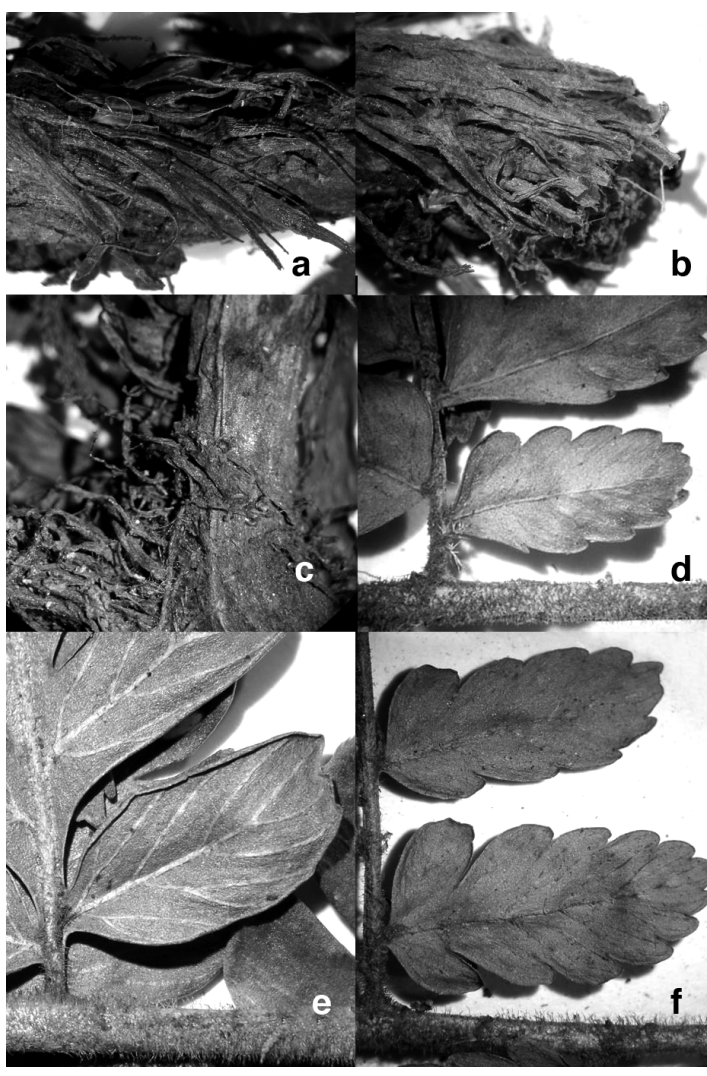

FIgURA 1. a, d. Polybotrya alfredii (A. Rojas 774, CR). a) Detalle del rizoma. d) Detalle de la lámina estéril. b, e. P. aureisquama (A. Rojas et al. 6986, CR). b) Detalle del rizoma. e) Detalle de la lámina estéril. c, f. P. lourteigiana (M. Jones \& P. Olivas 522, CR). c) Detalle del rizoma. f) Detalle de la lámina estéril.

Ramón, 10¹3'15”N, 94³6'00"W, 1 mar 1992, E. Schmidt 243 (CR). Cartago: Turrialba, Tayutic, Jicotea, Finca La Pradera, subiendo la Fila hacia San Antonio, 9 $47^{\circ} 15^{\prime \prime} \mathrm{N}, 83^{\circ} 33^{\prime} 15^{\prime \prime} \mathrm{W}, 1400 \mathrm{~m}, 14$ jun 1995, G. Herrera 7864 (CR); Cantón de Turrialba, Valle del Reventazón, Finca La Pradera, Jicotea, $9^{\circ} 47^{\prime} 15^{\prime}$ N, $83^{\circ} 32^{\prime} 35^{\prime}$ 'W, 1000 m, 22 jun 1995, G. Rivera \& A. Rojas 2486 (INB); Cantón de Turrialba, Cordillera de Talamanca, Tayutic, Jicotea, $9^{\circ} 47^{\prime} 15^{\prime}$ 'N, 83³2'50”'W, 1100-1600 m, 22 jun 1995, A. Rojas et al. 2029 (INB, MO). Guanacaste: Parque Nacional Guanacaste, Estación Pitilla, Fila Orosilito, $11^{\circ} 02^{\prime} \mathrm{N}$, 85'25.3'W, 100 m, 14 jun 1989, B. Hammel et al. 17451 (CR, MO); Cantón de La Cruz, Parque Nacional Guanacaste, Volcán Orosí, Estación Pitilla, $10^{\circ} 58^{\prime} 42^{\prime}$ N $, 85^{\circ} 25^{\prime} 49^{\prime \prime} \mathrm{W}, 700-800 \mathrm{~m}, 6$ jul 1998, $C$.

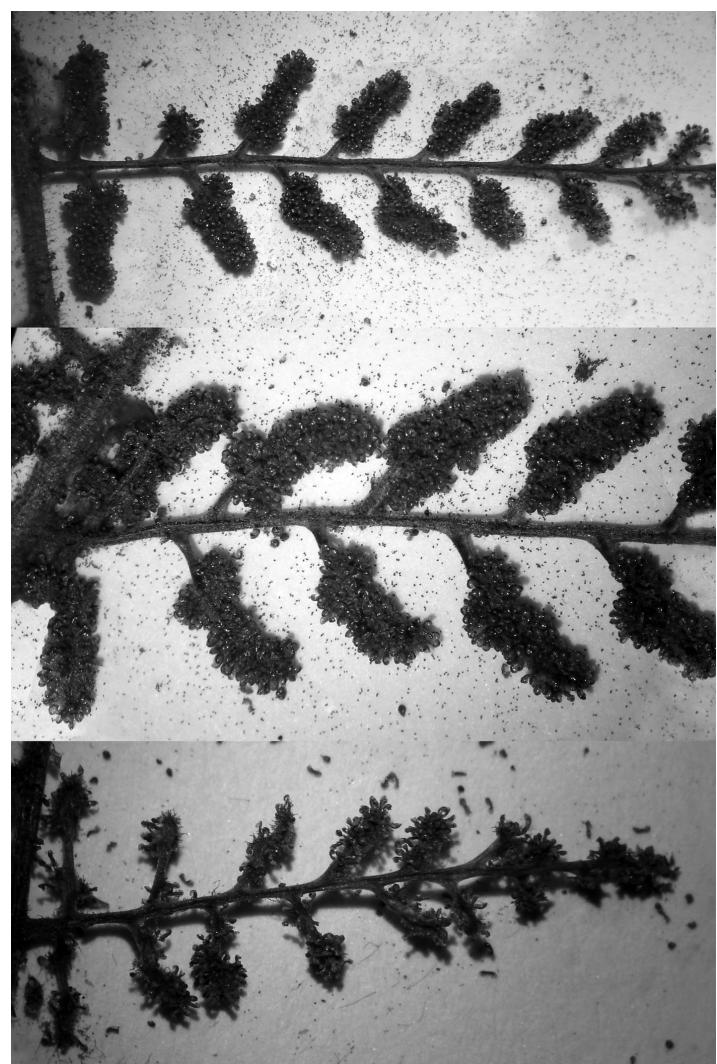

Figura 2. a. Polybotrya alfredii (A. Rojas 774, CR); detalle de la lámina fértil. b. P. aureisquama (A. Rojas et al. 6986, CR); detalle de la lámina fértil. c. P. lourteigiana (M. Jones \& P. Olivas 522, CR); detalle de la lámina fértil.

Moraga \& P. Ríos 1014 (CR, INB); Cantón de Tilarán, Cordillera de Tilarán, Río San Lucas, $10^{\circ} 22^{\prime} 50^{\prime} \mathrm{N}$, 8454'20”'W, 800-900 m, 27 jun 1995, G. Rodríguez \& A. Rojas 367 (CR, INB, MO); Cantón de Tilarán, Cordillera de Tilarán, Volcán Tenorio, Río San Lorenzo, 10³7’40”N, 8459'45”'W, 1050 m, 26 jul 1995, A. Rojas \& G. Rodríguez 2092 (INB). Heredia: Braulio Carrillo National Park, $10^{\circ} 20^{\prime} \mathrm{N}, 84^{\circ} 10^{\prime} \mathrm{W}$, 1000 m, 12 nov 1986, E. Hennipman et al. 6860 (CR), 1215 m, 13 nov 1986, E. Hennipman et al. 6887 (CR); vertiente Atlántica del Volcán Barva, transecto entre el Volcán Barva y Finca La Selva, 700 m, 2003, J. Kluge 2550 (USJ). Limón: Guápiles, borde de la Laguna Vianey Hernández, 25 abr 1986, I. Chacón \& A. Chacón 1871 (CR); Cantón de Talamanca, Parque Internacional La Amistad, Cordillera de Talamanca, 
antes de la Unión de Ríos Lori y Coén, entre Ujarrás y San José Cabecar, 9'24'20'W, 83¹3'30"W, 15002160 m, 3 abr 1993, A. Fernández 991 (CR). Puntarenas: Reserva Biológica Monteverde, Peñas Blancas, river valley, $10^{\circ} 19^{\prime} \mathrm{N}, 84^{\circ} 43^{\prime} \mathrm{W}, 800 \mathrm{~m}, 18$ mar 1989, W. Haber 9210 (CR). San José: Dota, Faldas del Cerro Nara, 9²9'40'N, 840'50'W, 800900 m, 14 abr 1998, A. Estrada \& O. Valverde 1535 (CR); Parque Nacional Braulio Carrillo, Estación Carrillo, sendero a tanques de agua, $700 \mathrm{~m}$, sin fecha, L. Gómez et al. 22914 (CR); Cantón de Vásquez de Coronado, Parque Nacional Braulio Carrillo, cuenca del Sarapiquí, Estación Carrillo, Quebrada Sanguijuela, 1009'38”N, 8357'23”'W, 700 m, 23 mar 1999, $A$. Rojas \& E. Jenkins 4996 (CR, INB).

Polybotrya aureisquama difiere de $P$. alfredii Brade porque las escamas del rizoma son más anchas (1.5$2.5 \mathrm{~mm}$ de ancho vs. $1.0-1.5 \mathrm{~mm}$ ), doradas a ferrugíneas (vs. pardas), flácidas (vs. rígidas) y con el margen ciliado (vs. entero), frondas más pequeñas [50-70 $(-85) \mathrm{cm}$ de largo vs. $85-115 \mathrm{~cm}$ ], costas y cóstulas con pelos más largos (0.5-1.0 mm de largo vs. 0.1-0.3 $\mathrm{mm}$ ) y tejido laminar poco diferenciado en color entre las dos superficies donde ambas son verde claro (vs. tejido laminar marcadamente diferenciado en color donde el haz es verde oscuro y el envés verde claro) (Figs. 1 y 2).

Etimología: El nombre de la especie hace referencia a las escamas del rizoma doradas.

\section{Polybotrya insularis A. Rojas, sp. nov.}

TIPO: Costa Rica. Puntarenas: Cantón de Puntarenas, Parque Nacional Isla del Coco, Isla del Coco, Bahía Wafer, alrededores y camino a Chatham, $5^{\circ} 32^{\prime} 5^{\prime} \mathrm{N}, 87^{\circ} 02^{\prime} 40^{\prime \prime} \mathrm{W}, 0-150 \mathrm{~m}, 17$ jun 1997, $A$. Rojas 3591 (holotipo: INB; isotipo: MO). FIG. 3.

A Polybotrya caudata Kunze frondibus sterilibus brevioribus, pilis rachi, costulis venisque sparsis, frondibus fertilibus bipinnatis differt.

Hemiepífitas; rizoma de 0.5-0.7 cm de diámetro, reptante; escamas del rizoma 3-7 x 0.5-1 mm, linear-lanceoladas, pardas, lustrosas, irregularmente dentadas; frondas estériles de 65-100 cm de largo; estípite 1/3$2 / 5$ del tamaño de la fronda, pajizo, escamoso, las escamas hasta $10 \mathrm{~mm}$ de largo, similar a las del rizoma

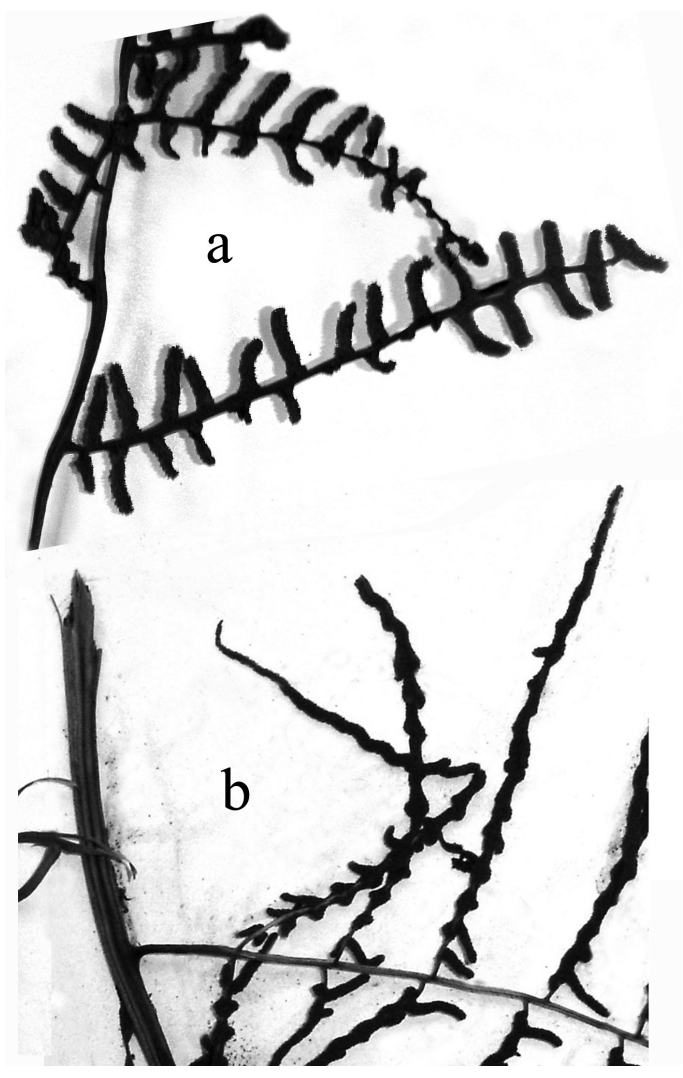

Figura 3. a. Polybotrya insularis (J. Trusty \& H. Kesler III 488, CR); detalle de la lámina fértil. b. P. caudata ( $A$. Rodríguez 847, CR); detalle de la lámina fértil.

pero restringidas a la base; lámina estéril 35-55 x 30-40 $\mathrm{cm}$, deltada, 2-pinnado-lobulada a 2-pinnado-pinnatífida, el tejido laminar glabro entre las nervaduras y el margen; pinnas 15-20 x 5-10 cm, anádromas; pínnulas de las pinnas medias $2-5 \times 0.5-2 \mathrm{~cm}$, fuertemente excavadas basiscópicamente; ejes glabrescentes, los tricomas 0.1-0.4 mm, aciculares, hialinos, patentes, dispersos; surcos dorsales generalmente dispersamente pelosos por dentro, más pelosos en las inserciones; nervaduras libres; frondas fértiles de $80-90 \mathrm{~cm}$ de largo estípite ca $1 / 2$ del tamaño de la fronda; lámina de 40-50 x 20-25 cm, oblongas, 2-pinnadas, las pínnulas lineares, enteras; esporas normalmente desarrolladas.

DistribuCión: En las bahías Wafer y Chatham en la Isla del Coco a 0-150 m.

Paratipos: COSTA RICA. Puntarenas: Cocos Island, high plateau SE of Chatham Bay, 9-11 abr 1979, $R$. Foster 4160 (CR); Cocos Island, Wafer, mar 1970, L. 
Gómez 3354 (CR); Isla Cocos, Valle de Wafer y sector NW de la isla, 0-100 m, mar 1974, L. Gómez 4528 (CR); Isla Cocos, región inferior del Valle Wafer, 0-100 m, mar 1974, L. Gómez 4537 (CR); Isla del Coco, Chatham Bay, 13 abr 1965, A. Jiménez 3200 (CR); Isla del Coco, 6-8 jun 1989, R. Soto s.n. (USJ); Cantón de Puntarenas, Parque Nacional Isla del Coco, $5^{\circ} 32^{\prime} \mathrm{N}$, $87^{\circ} 03^{\prime} \mathrm{W}$, nov 2002, J. Trusty \& H. Kesler III 488 (CR); Cantón de Puntarenas, Parque Nacional Isla del Coco, $5^{\circ} 32^{\prime} \mathrm{N}, 89^{\circ} 02^{\prime} \mathrm{W}$, nov 2002, J. Trusty 503 (CR); Isla del Coco, 4 mayo 1936, M. Valerio 2225 (CR).

A diferencia de Polybotrya caudata Kunze, la nueva especie hibridiza con P. polybotrioides (Baker) H. Christ, y los híbridos son fáciles de distinguir por su lámina estéril con división intermedia entre las dos especies. Aunque las primeras colecciones son conocidas desde 1965 (A. Jiménez 3200, CR), Moran (1987) no la menciona en su monografía del género e indica la ausencia de híbridos en Polybotrya. Especimenes adicionales conocidos del híbrido son: L. Gómez 4535 (CR), L. Gómez 18064 (CR), A. Rojas 3578 (INB) y J. Trusty \& H. Kesler III $488 a$ (CR).

Rojas y Trusty (2004) incluyeron a la nueva especie en Polybotrya osmundacea Humb. et Bonpl. ex Willd. por tener lámina estéril similar en división, pínnulas fuertemente excavadas basalmente, tejido laminar glabro, pelos de los ejes hialinos y patentes, pínnulas basales de las pinnas medias y basales anádromas; pero difiere por frondas estériles más pequeñas (65-100 cm de largo vs. 120-150 cm), ejes no escamosos (vs. escamosos) y dispersamente pilosos (vs. pelos medianamente densos), frondas fértiles oblongas (vs. ovadas) y bipinnadas (vs. tripinnadas a tetrapinnadas), como en el subgénero Soromanes (Fée) R.C. Moran.

Moran (1987) incluyó esta especie en Polybotrya caudata Kunze, de la cual difiere por frondas estériles más pequeñas $(65-100 \mathrm{~cm}$ de largo vs. $120-160 \mathrm{~cm})$, ejes glabrescentes (vs. por lo común densamente pilosos), tejido laminar glabro (vs. comúnmente peloso), pínnulas de las pinnas basales y medias anadrómicas (vs. catadrómicas) y lámina fértil oblonga (ovada o deltada) (Fig 3).

ETIMOLOGía: El nombre se refiere a la distribución de la especie, conocida sólo de la Isla del Coco en Costa Rica.
Polybotrya lourteigiana Lellinger, Proc. Biol. Soc. Wash. 89: 723. 1977.

TIPO: ColomBIA. Chocó, trail along ridge from the confluence of the forks of the Río Mutatá above the Río Dos Bocas to the top of Alto del Buey, ca. 14501750 m, D. Lellinger \& E. Sota 251 (Holotipo: US!; Isotipos: COL, LP).

Esta especie se puede separar de Polybotrya alfredii Brade, con la cual ha sido confundida en Costa Rica, por costa y cóstulas con pelos más largos (0.5-1.5 mm de largo vs. 0.1-0.3 mm), laxos (vs. rígidos) y hialinos a amarillentos y a menudo con la unión de células rojiza (vs. pardo clara), tejido laminar poco diferenciado en color entre las dos superficies donde ambas son verde claro (vs. tejido laminar marcadamente diferenciado en color donde el haz es verde oscuro y el envés es verde claro), lámina fértil 4-pinnada (vs. 3-pinnada) con los segmentos fértiles 1-2 mm de largo (vs. 2-4 $\mathrm{mm}$ ) y los terminales hasta $3 \mathrm{~mm}$ de largo (vs. $7 \mathrm{~mm}$ ). El material costarricense particularmente tiene lámina más dividida (Figs. 1 y 2).

\section{Distribución: Costa Rica y Colombia.}

MATERIAl DE NUEVA DistribUCiÓn: COSTA RICA. Alajuela: Arenal Volcano, devastated area, W-SWof Arenal Volcano, 16 jul 1987, U. Funk 10429 (CR), U. Funk 10436 (CR); Arenal Volcano, along trail to devastated zone from observatory road, 16 feb 1989, G. Russel et al. 717 (CR). Cartago: Turrialba, Parque Nacional Barbilla, Cuenca de Matina, sendero principal, junto al Río Dantas, 958'20”N, $83^{\circ} 27^{\prime} 10^{\prime \prime} \mathrm{W}, 300-400 \mathrm{~m}, 26$ jun 2000, E. Mora 1346 (INB); Turrialba, Reserva Biológica Barbilla, Cuenca del Matina, Sendero Las Quebradas, 957'48'N, $83^{\circ} 27^{\prime} 51^{\prime \prime} \mathrm{W}, 500 \mathrm{~m}, 8$ oct 1999, E. Mora \& E. Rojas 513 (INB). Heredia: Parque Nacional Braulio Carrillo, Estación Magsasay, 5 jul 1990, G. Carballo 147 (CR, MO); area between Río Peje and Río Sardinalito, Atlantic slope of Volcán Barva, $10^{\circ} 18.5^{\prime} \mathrm{N}, 84^{\circ} 04^{\prime} \mathrm{W}, 480-520 \mathrm{~m}, 8$ abr $1986, M$. Grayum 6879 (CR, MO); Sarapiquí, Puerto Viejo, Estación Biológica La Selva, $10^{\circ} 26^{\prime} \mathrm{N}, 84^{\circ} 01^{\prime} \mathrm{W}, 50$ m, 8 oct 2001, M. Jones \& P. Olivas 279 (CR), 18 oct 2001, M. Jones \& P. Olivas 298 (CR); 18 abr 2002, M. Jones \& P. Olivas 389 (CR), 5 Jul 2002, M. Jones $\&$ P. Olivas 510 (CR), 12 jul 2002, M. Jones \& P. 
Olivas 522 (CR). Limón: Cantón de Pococí, Parque Nacional Braulio Carrillo, Cuenca del Sarapiquí, Quebrada González, Sendero Las Palmas, $10^{\circ} 09^{\prime} 48^{\prime \prime} \mathrm{N}, 83^{\circ} 56^{\prime} 20^{\prime \prime} \mathrm{W}, 500 \mathrm{~m}, 6$ nov 1997, $A$. Rodríguez \& L. Vargas 2688 (INB).

Agradecimientos. Agradezco a los herbarios del Museo Nacional de Costa Rica (CR), Instituto Nacional de Biodiversidad (INB) y Missouri Botanical Garden (MO) por permitirme utilizar sus colecciones, a Hamer Salazar por ayudarme en la logística para hacer investigaciones en la Reserva Biológica Alberto M. Brenes, a Cyril Nelson por la traducción de las diagnosis al latín y a los revisores anónimos por sus comentarios que mejoraron la calidad de este estudio.

\section{LITERATURA CITADA}

Lellinger, D.B. 1972. Five new species of South American Polybotrya. Amer. Fern J. 62: 49-56.

Lellinger, D.B. 1977. Nomenclatural and taxonomic notes on the Pteridophytes of Costa Rica, Panama and Colombia, I. Proc. Biol. Soc. Wash. 89: 7703-732.

Moran, R.C. 1987. Monograph of the neotropical ferns genus Polybotrya (Dryopteridaceae). III. Nat. Hist. Survey Bull. 34: 1-138.

Moran, R.C. 1995. Polybotrya, p. 216-218. In R. C. Moran \& R. Riba (eds.). Flora Mesoamericana. Volumen 1. Psilotaceae a Salviniaceae. Univ. Nac. Autónoma de México. México D. F., México.

Rojas, A.F. y J. Trusty. 2004. Diversidad Pteridofítica de la Isla del Coco. Brenesia 62: 1-14.

Tryon, R.M. y R.G. Stolze. 1991. Pteridophyta of Perú. Part IV. 17. Dryopteridaceae. Fieldiana, Bot, n.s. 27: 176 p. 